\title{
Design of a Broadcasting Modem for a DC PLC Scheme
}

\author{
Eric R. Wade, Student Member, IEEE, and Haruhiko Harry Asada, Member, IEEE
}

\begin{abstract}
A new type of power line carrier communication (PLC) technique is developed to reduce cable requirements for robotic and vehicular systems. An electrical line connecting a dc power supply to motor drives and sensor units is used for transmitting data as well as for delivering the dc power. Unlike conventional ac power line communication, the dc power bus has predictable noise and impedance characteristics that allow for large fanout and high bandwidth. First, the basic architecture of the dc power line communication is presented. Then, a design of a high-fanout modem that uses a Guanella-type transmission line transformer (TLT) is presented. The serial windings and special resonance characteristics of the TLT coupled with a capacitor are exploited to minimize attenuation in high-fanout systems. The modem characteristics including high-frequency parasitic dynamics are analyzed, and the end-to-end line impedance and signal gain are evaluated as the number of nodes goes to infinity. A prototype dc power line communication system is then built, tested, and applied to a design for modular reconfigurable robots.
\end{abstract}

Index Terms-DC power systems, power line communication (PLC), reconfigurable architectures, sensor network.

\section{INTRODUCTION}

$\mathbf{T}$ HE number of actuators and sensors used in robotic systems, automobiles, and manufacturing equipment keeps increasing. A humanoid robot, such as Honda's ASIMO, has 26 actuators and multiple sensors for its head, limbs, and joints. ${ }^{1}$ A standard machining center can contain more than ten actuators and 50 sensors, while current high-end automobiles have over 200 actuators and at least as many sensors. The design of a standard servoed axis requires two cable harnesses connecting each actuator to its driver and controller; one is a signal harness for the encoder and other sensors, and the other is a power harness for delivering high-voltage (or current) power to the actuator. As the number of degrees of freedom (DOFs) increases, these cables create major problems. Special consideration must be made for any node located through a bending or rotating joint to prevent arcing or fraying of the cables. Furthermore, power cable lines between an actuator and its drive amplifier create electromagnetic interference (EMI) and noise problems. Additionally, cabling is extremely costly. For instance, in automobiles, a pound of cabling can cost as much as \$3-6\$ in design

Manuscript received January 13, 2006; revised June 12, 2006. Recommended by Technical Editor C. Maviroidis. This work was supported in part by the National Science Foundation under Grant 0413242.

The authors are with the Department of Mechanical Engineering, Massachusetts Institute of Technology, Cambridge, MA 02139 USA (e-mail: erwade@mit.edu; asada@mit.edu).

Digital Object Identifier 10.1109/TMECH.2006.882983

\footnotetext{
${ }^{1}$ Inside Asimo (Honda Website). [Online]. Available: http://asimo.honda. com/inside_asimo.asp?bhcp $=1$
}

costs [1]. Finally, the complex routing requirements often preclude automated installation and inhibit maintenance.

Reducing cables has been of interest to many industries, resulting in methods for replacing serial data lines by a parallel line. Various standards have been established for such data lines [2]. Although these bus lines reduce the cable thickness, two separate cables are still required for signal and power transmissions in each servoed axis.

Research on in-home networking has led to power line carrier communication (PLC), which consolidates power and data transmission by sending data over already existing power lines. It reduces cabling requirements, and has been shown to be a useful and economically viable technology in the recent years with methods utilizing digital modulation techniques to achieve data rates of up to $1 \mathrm{Mb} / \mathrm{s}$ [3]-[6].

However, PLC has a number of limitations. The large power transformers that are used to step down power from outdoor power lines to individual houses attenuate high-frequency signals required for broadband communication [4]. Further, residential loads such as dimmers and switching power supplies often contribute noise in ranges anywhere between $100 \mathrm{~Hz}$ and $1 \mathrm{MHz}$ [5]. Due to load uncertainty, impedance, noise, and signal attenuation vary over a wide range and are highly complex and nonlinear.

Another problem for PLC is bandwidth limitations due to regulation. The Federal Communications Commission (FCC) in the United States limits power line transmissions to the $10-450-\mathrm{kHz}$ range, while the European Commission for Electrotechnical Standardization (CENELEC) currently confines transmission to the $3-148.5-\mathrm{kHz}$ range, which severely limits the bit transmission rate for PLC [7], [8]. Communication outside of these ranges is shared by many other operators such as amateur broadcasters. ${ }^{2}$ Thus, traditional PLC has numerous difficulties that have limited its success so far.

In this paper, an alternative architecture will be developed for vast DOF robotic systems and other electromechanical systems. Instead of using an ac power line, signals are transmitted over a de power bus connecting a dc power supply to various nodes. This dc PLC scheme, along with proper modem design, allows us to connect numerous actuators and sensors. In the following sections, we introduce a new PLC architecture, and discuss its feasibility and strengths. We then design a data modem, and build and test a prototype system. Finally, we apply the system to a reconfigurable modular robotic system, where both power and signal lines are replaced by a single pair of wires, facilitating modular design.

${ }^{2}$ FCC Bandwidth Allocation Chart. [Online]. Available: http://www.ntia.doc. gov/osmhome/allochrt.pdf 


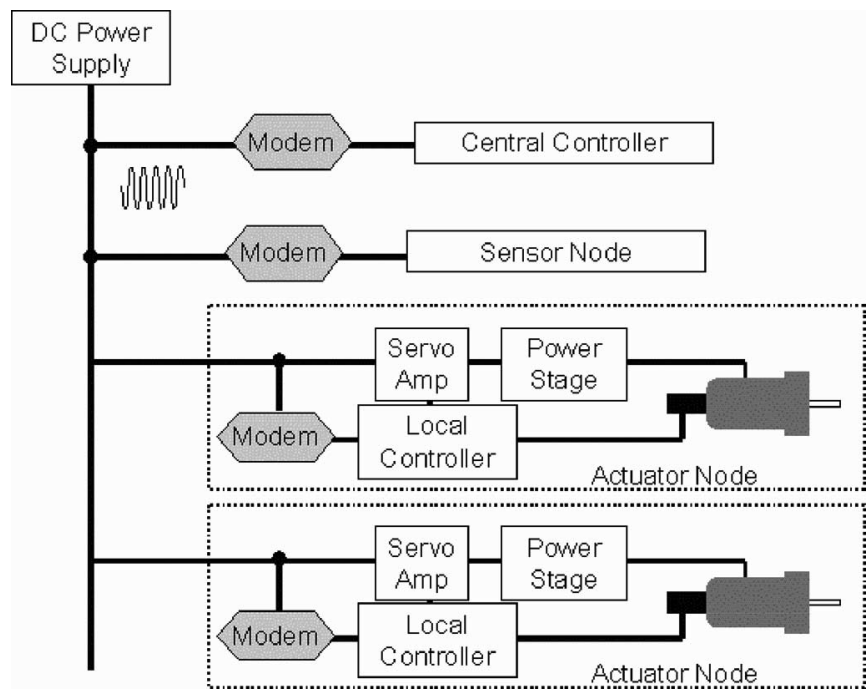

Fig. 1. Architecture of the dc power bus communication system.

\section{DC PLC NETWORK}

\section{A. Architecture}

Our PLC architecture is intended to apply to a confined, local system where the dc power is supplied to all of the nodes. Such closed environments with shared dc power can be found in robotic systems, vehicles, and manufacturing equipment. As shown in Fig. 1, a single power bus line originating with a dc power supply extends to a number of "nodes," or actuator and sensor units. Encoder readings, sensor signals, and control commands, are coded, modulated, and superimposed on the dc power line. The single dc voltage line, that we term the consolidated power/signal (CPS) line and a ground line are used for connecting the nodes.

Note that, unlike traditional actuator drive systems, where drive amplifiers are placed inside a control console and long cables are used for connecting the actuators to the drives, this dc power bus network utilizes distributed drives placed near the actuators. As shown in Fig. 1, a power stage and a local controller are placed near the actuator, leaving long-distance power and data transmission to the single CPS line. The so-called integrated motors also have their servo amplifiers housed inside the actuator body [9]. In the proposed system, the servo amplifier does not have to be inside the actuator body, but can be placed anywhere between the actuator and a dc power supply. Since the cables between the actuator and the drive are in general highvoltage, high- $d V / d t$ lines, reducing the distance of such cables will effectively reduce electromagnetic interference (EMI) and ground noise.

This dc PLC technique has a few more important features. The dc power supply acts as a buffer between the dc bus line and loads on the ac mains. Because of this, the line's characteristics do not depend on those loads beyond the dc power supply. Furthermore, since no large inductance transformers are directly connected to the line, the line impedance at high frequencies is much higher than that of the traditional ac power lines. Another important advantage of the dc PLC is that the load conditions are predictable. Modems for transmitting signals can be designed

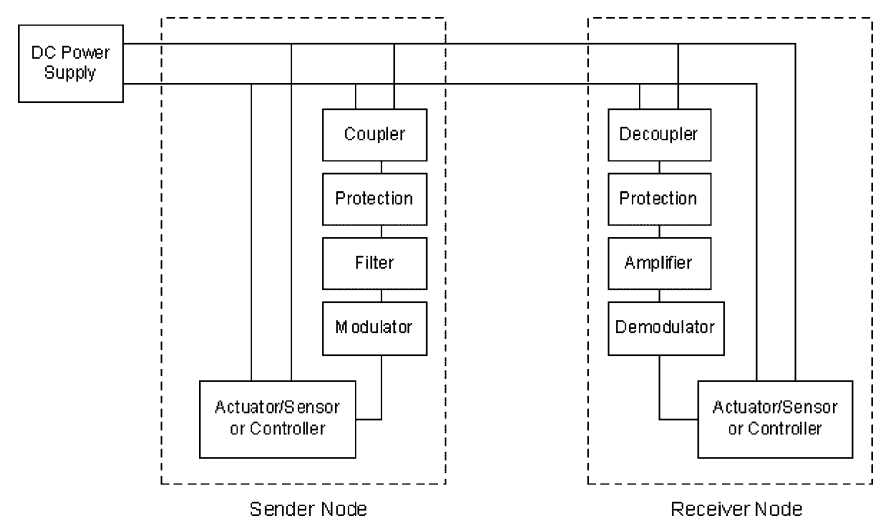

Fig. 2. Block diagram of the dc bus transmission components.

based on a priori characterization of loads and noise sources. This allows for the reliable broadband communication needed for multiaxis servo control.

\section{B. Technical Issues}

The above architecture may have numerous variations and diverse levels of sophistication. In this paper, however, a focus will be placed on the basic physical layer and hardware modem design suitable for the dc PLC. In designing such a physical layer, an immediate concern of the technology is to what extent the dc bus can be used as a communication medium. Interferences with the dc power supply might make the power line communication infeasible or difficult. There are two possible failure scenarios that must be considered. The first is severe attenuation of the data signal.

There is a possibility that the low impedance of the dc power supply will draw too much signal current and cause attenuation of the data signal. Much work has been done to investigate this problem in ac power lines [3], [4]. However, little data is available on the dc power lines, which is fundamentally different from the ac power line.

The second possible failure scenario is data corruption due to the switching noise created by active devices. We must ensure that these adverse effects of using the dc bus can be suppressed in the frequency ranges in which we will operate. These failure scenarios are investigated in Section IV.

\section{Modem Design AND ANalysis}

\section{A. Hardware Requirements and Problems}

Given that the dc power line can be used as a communication medium, we now describe a method for fully exploiting the features of the dc PLC technique. Specifically, we are interested in networking a large number of actuators and sensors. There are two significant technical challenges; minimizing signal attenuation and maintaining sufficient line impedance to keep the driving current low even when large numbers of nodes are connected to the CPS line. In this section, we present a modem that achieves these goals.

Fig. 2 shows the major components involved in the sender and receiver nodes. The modulator, demodulator, amplifiers, 


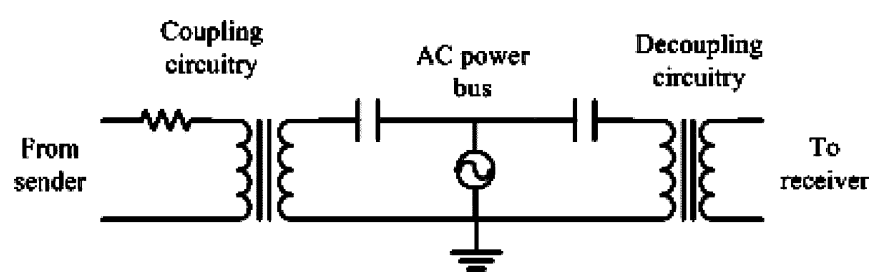

Fig. 3. Standard coupling/decoupling circuitry.

and filters are all standard modem components. However, as this modem is for use in a PLC system, additional protection, coupling, and decoupling components are required. The primary functional requirement for these components is the ability to superimpose the data signal onto the dc power line. Additionally, we must be able to block the dc voltage and current to prevent saturation of and damage to the modem.

Fig. 3 shows a standard coupling and decoupling circuit used for ac power line communication [10]. The transformer electrically isolates the data and power sources. The capacitor is used to block the dc power from the modem transmit- and receivecomponents. The transformer and capacitor provide a path for the ac data signal to be superimposed on the dc power line. This standard circuit, however, does not meet our requirements due to the parasitic effects of the transformer at high frequencies. Parasitics such as winding resistance and magnetizing inductance, typically ignored in the ideal transformer model, cannot be neglected for our high-frequency application. These parasitics, which are in parallel with the windings, cause insertion losses. These resonances detract from ideal behavior and draw nonnegligible amounts of current. This current flows from the supply line directly to the ground, and never passes to the load, causing substantial attenuation of the data signal. As the number of nodes increases, this attenuation can critically reduce the data signal level on the CPS until it is too low to drive the receiver nodes.

\section{B. Use of Transmission Line Transformer (TLT)}

To minimize the attenuation associated with the coupling and decoupling circuits, we utilize TLTs, which have substantially lower insertion losses than traditional transformers [11], [12]. A major advantage of TLTs is that parasitics associated with the transformer are in series with the load. Even when these parasitics draw nonnegligible current, the current is ultimately delivered to the load. Thus, the TLT is less susceptible to parasitic losses than the standard transformers.

The impedance transformation afforded by the TLT will allow us to ensure that the power supply input impedance, $Z_{\mathrm{ps}}(s)$, appears much higher than that of the receivers, $Z_{r}(s)$. The system schematic, including the Guanella TLT in a 1:4 impedance transformation configuration, is shown in Fig. 4. Note that the TLTs at the sender and receiver sides are symmetric to one another with respect to the CPS line.

TLTs are available from multiple vendors. For the engineer without the time or resources to build a custom TLT, we will later outline a procedure by which the ideal TLT parameters can be found. Subsequently, the user can select an off-the-shelf TLT

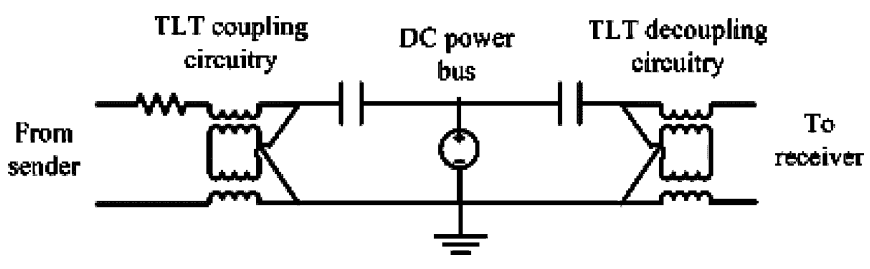

Fig. 4. DC PLC coupling/decoupling circuit.

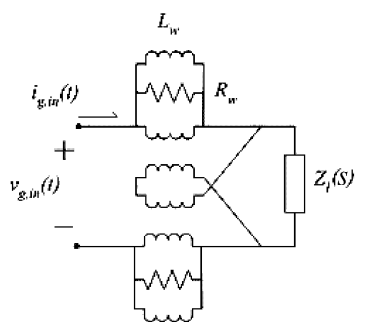

Fig. 5. Guanella TLT model accounting for magnetizing inductance and winding resistance.

that best meets the requirements. However, for the purposes of modeling the system, we will first assume that we have the ability to tune our own TLT according to the methods described in [11]. Our design requirements for the tuning of these parameters will result from a careful analysis of the TLT functionality.

\section{TLT Modeling}

The TLT model we use is based on that presented by Kuo et al. [12], which incorporates the magnetizing inductance and winding resistance of the transformer and can predict both the low- and high-frequency responses of the TLT. The difference in our formulation is due to the fact that a basic 1:1 isolating transformer is modeled in [12], whereas a 1:4 Guanella-style transformer is needed in this work. Nonetheless, the applicability of Kuo et al.'s model to our Guanella TLT is verified by close agreement between the experimental and the model results. Based on this model, the ideal TLT circuit is redrawn in Fig. 5 to explicitly include the winding inductance $L_{\mathrm{w}}$, and the winding resistance $R_{\mathrm{w}}$. Let $i_{\mathrm{g}, \text { in }}(t)$ and $v_{\mathrm{g}, \text { in }}(t)$ be the Guanella input current and voltage, respectively, and $Z_{1}(s)$ the equivalent load resistance placed across the output terminals. Replacing the winding inductance $L_{\mathrm{w}}$ with the magnetizing inductance, $L_{\mathrm{m}}=2 L_{\mathrm{w}}$, and applying impedance-based modeling techniques, the TLT impedance $Z_{\mathrm{t}}(s)$ seen at the input terminals can be written as

$$
Z_{\mathrm{t}}(s)=\frac{V_{\mathrm{g}, \text { in }}(s)}{I_{\mathrm{g}, \text { in }}(s)}=\left(\frac{8 R_{\mathrm{w}} Z_{1}}{Z_{1}+2 R_{\mathrm{w}}}\right) \frac{s}{s+\left[\frac{2}{L_{\mathrm{m}}}\left(\frac{R_{\mathrm{w}} Z_{1}}{Z_{1}+2 R_{\mathrm{w}}}\right)\right]} .
$$

See Appendix A for derivation.

We experimentally determine the values for $L_{\mathrm{m}}$ and $R_{\mathrm{w}}$ using an HP4194A impedance analyzer with a bandwidth of 40MHz. The TLT was terminated with various loads to obtain experimental values for the parasitic components, which were used to solve (1). The magnetizing inductance $5 \mu \mathrm{H}$, and the winding resistance $450 \Omega$, were used along with various resistive terminations to produce the results in Fig. 6, which show close correlation between the experimental and the model performance. 


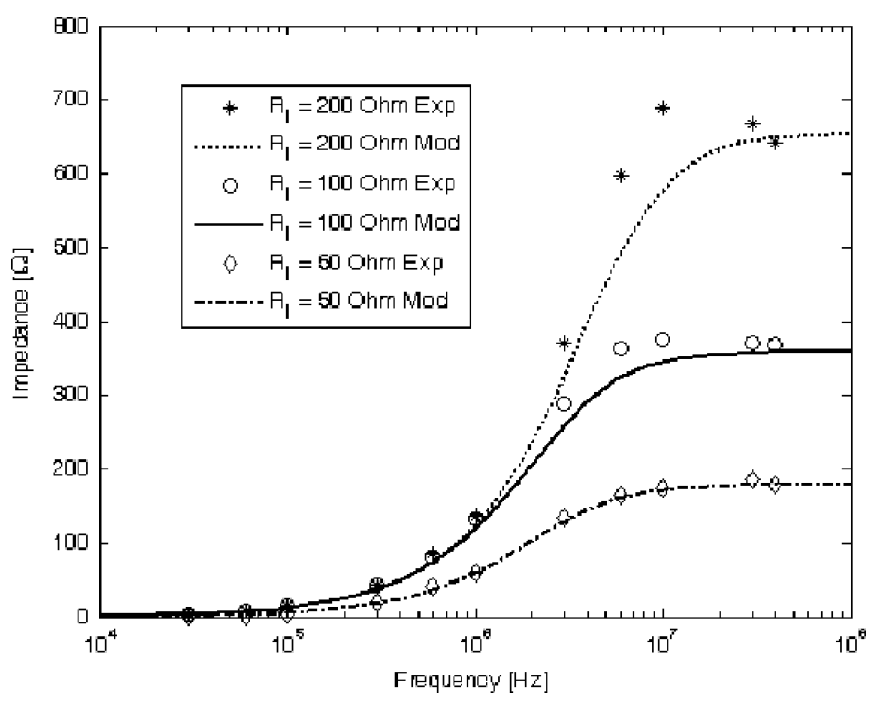

Fig. 6. Impedance of the TLT with a terminating resistor.

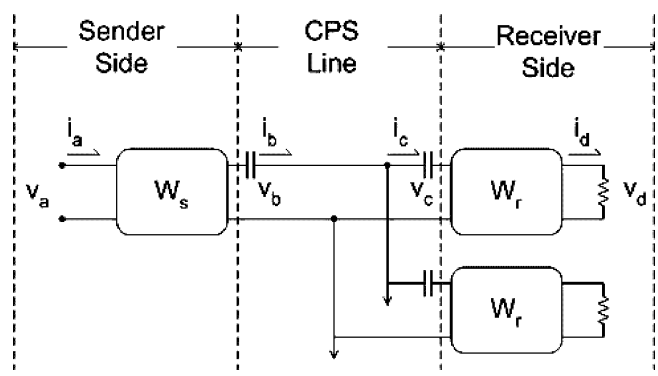

Fig. 7. Circuit divided into sections: sender-side components, CPS, and receiver-side components.

\section{System Performance}

Having verified the TLT model, the next step is to evaluate the entire system performance when multiple nodes are connected to the CPS line (see Fig. 7). To characterize impedance and gain, we note that the impedance seen from the sender-side terminals is given by

$$
Z_{n}(s)=\frac{V_{\mathrm{a}}(s)}{I_{\mathrm{a}}(s)}
$$

and the gain is given by

$$
G_{n}(s)=\frac{V_{\mathrm{d}}(s)}{V_{\mathrm{a}}(s)}
$$

The sinusoidal voltage input $V_{\mathrm{a}}(s)$ is generated by the sender and $V_{\mathrm{d}}(s)$ is the voltage observed at one of the receivers at steady state. $I_{\mathrm{a}}(s)$ is the sender-side current associated with the voltage $V_{\mathrm{a}}(s)$.

To obtain (2) and (3), it is convenient to use a $2 \times 2$ matrix formulation relating input current and voltage to output current and voltage. This allows us to evaluate the system characteristics independent of the loads and on a component-by-component basis. As shown in Fig. 7, the whole system can be divided into the sender TLT, the receiver TLT, and the CPS line (including the blocking capacitors). We use the derivation of (1) to write the equations of the sender-side TLT in $2 \times 2$ matrix form as

$$
\left[\begin{array}{c}
V_{\mathrm{b}} \\
I_{\mathrm{b}}
\end{array}\right]=\left[W_{\mathrm{s}}\right]\left[\begin{array}{c}
V_{\mathrm{a}} \\
I_{\mathrm{a}}
\end{array}\right]
$$

where

$$
W_{\mathrm{s}}=\left[\begin{array}{cc}
\frac{1}{2} & 0 \\
-\left(\frac{1}{4 R_{\mathrm{w}}}+\frac{1}{2 L_{\mathrm{m}} s}\right) & 2
\end{array}\right] .
$$

Similarly, the impedance of the receiver-side TLT can be used to write

$$
\left[\begin{array}{c}
V_{\mathrm{d}} \\
I_{\mathrm{d}}
\end{array}\right]=\left[W_{\mathrm{r}}\right]\left[\begin{array}{c}
V_{\mathrm{c}} \\
I_{\mathrm{c}}
\end{array}\right]
$$

where

$$
W_{\mathrm{r}}=\left[\begin{array}{cc}
2 & 0 \\
\left(\frac{1}{4 R_{\mathrm{w}}}+\frac{1}{2 L_{\mathrm{m}} s}\right) & \frac{1}{2}
\end{array}\right] .
$$

The current flowing to each receiver at steady state is $I_{\mathrm{c}}(s)$. Assuming that all the blocking capacitors in the sender and receiver modems are the same, we can relate the receiver-side voltage and current to those of the sender-side as

$$
\left[\begin{array}{c}
V_{\mathrm{c}} \\
I_{\mathrm{c}}
\end{array}\right]=\left[W_{\text {line }}\right]\left[\begin{array}{l}
V_{\mathrm{b}} \\
I_{\mathrm{b}}
\end{array}\right]
$$

where

$$
W_{\text {line }}=\left[\begin{array}{cc}
1 & -\left(1+\frac{1}{n}\right) \frac{1}{s C} \\
0 & \frac{1}{n}
\end{array}\right] .
$$

Combining (4), (6), and (8) gives

$$
\left[\begin{array}{c}
V_{\mathrm{d}} \\
I_{\mathrm{d}}
\end{array}\right]=\left[W_{\mathrm{r}}\right]\left[W_{\text {line }}\right]\left[W_{\mathrm{s}}\right]\left[\begin{array}{c}
V_{\mathrm{a}} \\
I_{\mathrm{a}}
\end{array}\right] .
$$

Solving this for (2) subject to the load resistance $V_{d}=R_{d} / I_{d}$ yields

$$
Z_{n}(s)=\frac{A s^{3}+B s^{2}+C s}{D s^{3}+E s^{2}+F s+G}
$$

where $Z_{n}(s)$ is the total system impedance. (See Appendix B for coefficients.)

As the number of nodes $n$ tends to infinity, the impedance function reduces to

$$
Z_{\infty}(s)=n \stackrel{\lim _{\rightarrow}}{\rightarrow} Z_{n}(s)=\frac{b_{1} s^{2}+c_{1} s}{d_{1} s^{3}+e_{1} s^{2}+f_{1} s+g_{1}} .
$$

These coefficients are dependent on two effective time constants, $\tau_{1}=R_{\mathrm{w}} C$ and $\tau_{2}=\sqrt{L_{\mathrm{m}} C}$. Typically, the resistance order of magnitude is hundreds of ohms, whereas the capacitor is $10^{-9} \mathrm{~F}$, and the inductance is $10^{-6} \mathrm{H}$. Thus, the time constant $\tau_{1}$ is of the order $10^{-7} \mathrm{~s}$ and $\tau_{2}$ is of the order $10^{-7.5} \mathrm{~s}$. We use these values to find $b_{1} \ll c_{1}$ and $d_{1} \ll e_{1}, f_{1}, g_{1}$, and to reduce $Z_{\infty}(s)$ to

$$
Z_{\infty}(s) \cong \frac{c_{1} s}{e_{1} s^{2}+f_{1} s+g_{1}}
$$




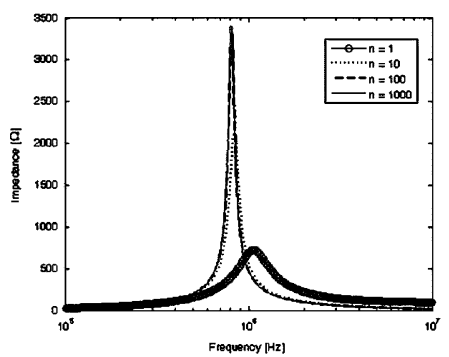

(a)

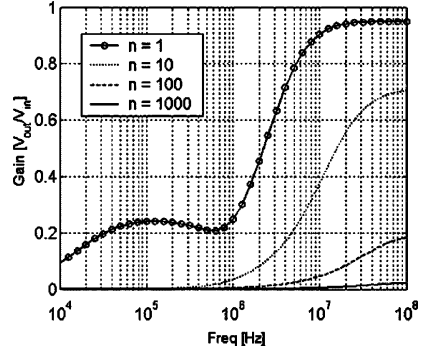

(b)
Fig. 8. Impedance and gain as a function of the number of receivers $(n)$.

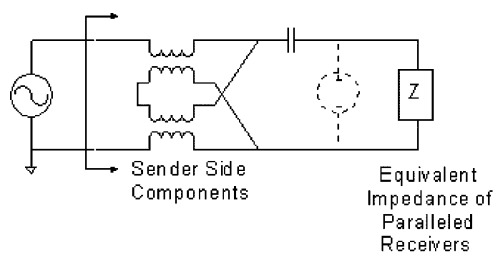

(a)

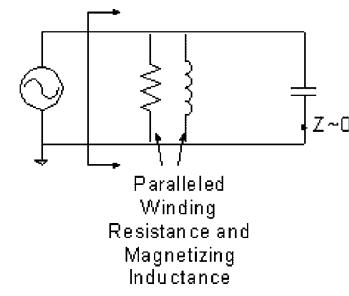

(b)
Fig. 9. Equivalent circuit model for large numbers of receivers $(n)$.

Similarly, the gain function $G(s)$ for $n$ receivers is given by

$$
G_{n}(s)=\frac{\alpha s^{3}}{\delta s^{3}+\gamma s^{2}+\varsigma s+\varepsilon} .
$$

(See Appendix C for coefficients.)

As $n$ approaches infinity, the gain function reduces to

$$
G_{\infty}(s)=n \stackrel{\lim }{\rightarrow} \infty G_{n}(s)=0 .
$$

The fact that $Z_{\infty}(s)$ goes to a constant value and that $G_{\infty}$ goes to zero makes logical sense. As $n$ goes to infinity (and the load resistance goes to zero), the impedance seen from the sender side will just be a function of the sender-side components. Similarly, when the load resistance goes to zero, the output voltage goes to zero, and thus, the gain goes to zero.

It is of interest to evaluate the role of $n$ in these relationships. The plot in Fig. 8(a) shows the frequency-dependent impedance for varying numbers of receivers $n$, which has a very distinct peak. Specifically, as the number of nodes increases, the peak amplitude increases, the peak frequency decreases, and the impedance at high frequencies decreases. It should be noted that, as the number of nodes tends to infinity, the impedance peak amplitude becomes extremely high.

The physical sense of this peaking behavior can be explained with Fig. 9. Recall the impedance form of (13). The peak frequency for this simplified expression is given by $\omega_{\mathrm{p}}=\sqrt{g_{1} / e_{1}}$. Referring to the parameters $e_{1}$ and $g_{1}$ from the appendix reveals that the value can be approximated by a constant multiplied by $1 / \sqrt{L_{\mathrm{m}} C}=1 / \tau_{2}$. This implies the resonant behavior of a parallel LC circuit.

As more loads are placed in parallel, the equivalent load impedance approaches zero. This effectively changes the circuit of Fig. 9(a) to that of Fig. 9(b). The coupling capacitor is in parallel with the winding resistance and magnetizing induc- tance of the TLT. The impedance function of this parallel RLC circuit matches the form of the impedance function $Z_{\infty}(s)$. In the equivalent RLC circuit, current must always pass through the receiver impedance as well as the coupling capacitor. Once again, the use of the TLT ensures that the current will always flow into the load. Note that the typical transformer orientation of Fig. 3 allows the current to flow back to the source without passing through the load. Thanks to the TLT, we are able to manipulate the impedance values even for large gain and large numbers of actuators $n$.

\section{E. High-Fanout Design Protocol}

This implies that the proposed modem design using a TLT will allow us to transmit signals to a large number of nodes simultaneously without drastically lowering the output impedance [see Fig. 8(a)]. Even for large values of $n$, there remains a regime over which the impedance is high. Typically, when fanout becomes large, the impedance goes to zero, and infinite current is required to drive the system. However, in our apparatus, when fanout becomes large, there is always a frequency range for which only a nominal amount of current is required to drive the system. Of course, this frequency range is only useful if the gain of the transfer function $G_{n}(s)$, is not too small. Specifically, to use this high impedance value, we must ensure that the dip that is apparent in the gain curve of Fig. 8(b) is separable from the peak in the impedance curve.

Equation (14) indicates that the dip frequency is governed by the load resistance $R_{\mathrm{d}}$, and the coupling capacitance $C$. As mentioned earlier, the peak is a function of the time constant $\tau_{1}$. Thus, proper tuning of the $L_{\mathrm{m}}, C$, and $R_{\mathrm{d}}$ parameters ensures the separability of the impedance peak and the gain dip. This was verified experimentally through a set of tests in which the value of magnetizing inductance was changed from 0.5 to 5 to $50 \mu \mathrm{H}$. Thus, tuning the TLT components allows us to separate the impedance peak and the gain dip, which in turn allows us to broadcast information to multiple receivers simultaneously.

\section{IMPLEMENTATION AND EXPERIMENTS}

\section{A. Prototype}

A proof-of-concept prototype system has been developed to demonstrate the proposed approach and verify the design and analysis methods. The authors constructed a prototype dc PLC system to connect 30 actuator nodes located at five separate branches of the CPS line. The modem used at each node of the system consists of a TLT, a coupling capacitor, a microprocessor (PIC16F84), a signal modulation chipset, and a dc/dc converter. (A circuit diagram can be obtained from the authors directly.) The CPS line originates at the central controller and branches out to the four extremities, i.e., two arms and two legs, as well as to a central point within the humanoid apparatus. The dc power supply is a switching $12 \mathrm{VDC}$ regulator. The data transmission rate of $100 \mathrm{~kb} / \mathrm{s}$ is adequate to transmit 16-bit reference position data to all 30 local controllers of the integrated motors every $5 \mathrm{~ms}$. 


\section{B. Design}

Critical to this design is the selection of the transmission frequency and modem components. Experiments using the prototype will be described to verify the theoretical results and demonstrate the feasibility of the method.

1) Power Supply and Motor Characteristics: We begin with experiments for quantifying the system impedance and noise characteristics. We measured the impedance characteristics of fixed-voltage and bench-top dc power supplies using the HP4194A impedance analyzer with appropriate protection circuitry. At frequencies above $100 \mathrm{kHz}$, the dc power supply terminals have very high resistive impedance (above $4 \mathrm{k} \Omega$. This is due to the high-frequency impedance of the power supply output inductance.

To find the total line impedance, including the motors and drives, we used a protected milli-Ohm meter to measure the resistive impedance across the terminals of the motor and driver. The impedance is over $700 \Omega$ in the frequency range between $100 \mathrm{kHz}$ and $10 \mathrm{MHz}$. Thus, as long as the carrier frequency of transmitted signal is above a certain threshold, the dc power supply and motors should not cause significant attenuation.

The noise spectrum was measured with an oscilloscope with a sampling rate of $200 \mathrm{G}$-samples/s. There are two peaks in the noise spectrum at approximately $380 \mathrm{kHz}$ and in the 700$800-\mathrm{kHz}$ range. The noise magnitude is less than $-80 \mathrm{dBV}$ in the higher frequency range over $1 \mathrm{MHz}$. Even when ten motors were switched on and off randomly, the noise magnitude remains lower than $-43 \mathrm{dBV}$ in the megahertz range. Thus, for a signal with amplitude equal to $5 \mathrm{~V}$, the signal-to-noise ratio (SNR) is 56.9. This is sufficiently high that the noise should not drastically affect performance.

Of further interest to the reader might be the question of how the noise of the nodes in the system affects the CPS line characteristic. This is an interesting issue that has been addressed in various systems such as cellular phone networks, in-home power line communication networks, and automotive applications [13], [14]. Nonetheless, for the purposes of this paper, the authors will focus on the physical layer analysis.

2) Selection of Coupling Circuit Component Values: As mentioned earlier, there are many modulation techniques that can be used. To test our system, we use amplitude modulation (AM). ${ }^{3}$ We design the coupling circuitry based on the analysis described in the previous sections, frequency restrictions imposed by the power supply and motors, and filtering and impedance matching conditions. The design parameters are the transmission carrier frequency $f$, the coupling capacitance $C$, and the receiver load impedance $Z_{1}(s)$. In addition, we have the TLT impedance $Z_{\mathrm{t}}(s)$, which is a function of $L_{\mathrm{m}}$ and $R_{\mathrm{w}}$. The design procedure is as follows.

To determine the desired values for the TLT parameters $L_{\mathrm{m}}$ and $R_{\mathrm{w}}$, we treat the TLT as a high-pass filter. The cutoff frequency is a function of these two parameters and should be tuned such that only transmission frequencies (i.e., the mega-

\footnotetext{
${ }^{3}$ Carrier frequency values of AM chip sets have much more variability than their FSK counterparts. We chose to use AM in order to evaluate the transmission characteristics for a range of carrier frequencies from 2-10 MHz.
}

TABLE I

COUPLING CIRCUT PARAMETER VALUES

\begin{tabular}{|c|c|}
\hline Parameter & Value \\
\hline$C$ & $3.9 \mathrm{nF}$ \\
\hline$L_{m}$ & $5.1 \mu \mathrm{H}$ \\
\hline$R_{w}$ & $450 \Omega$ \\
\hline
\end{tabular}

Transmitted Signal
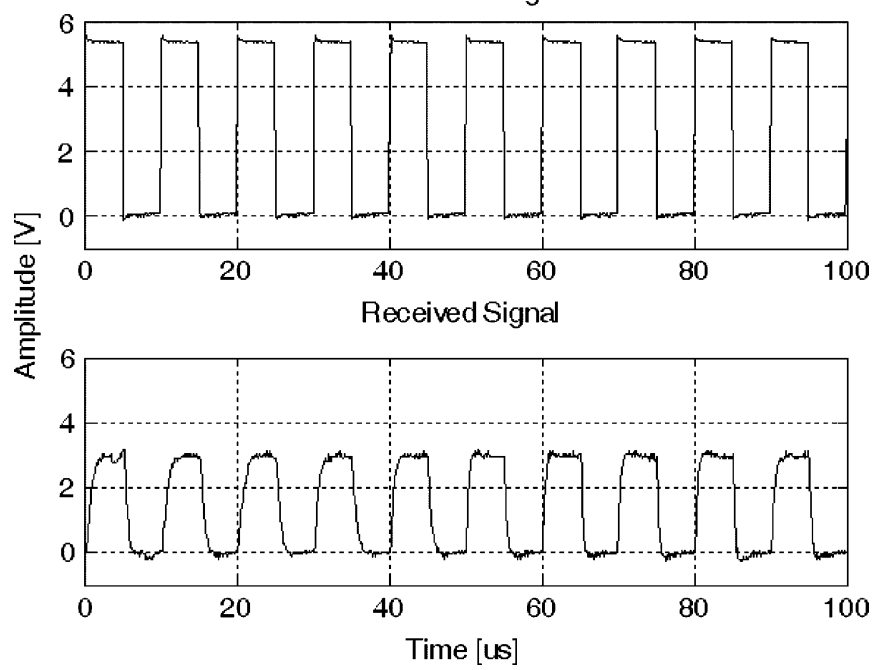

Fig. 10. Original transmitted digital signal and the received signal.

hertz range as mentioned in the previous section) can pass to the CPS. Thus, the frequency $f$ is found based on the desired transmission rate. This is used to select $R_{\mathrm{w}}$ and $L_{\mathrm{m}}$. We determine $Z_{\mathrm{c}}(s)$ by evaluating the operating current and voltage for the signal-processing components.

The first step after receiving the signal is to demodulate it. At the demodulator, the signal is divided between two ports that can sink a total of $250 \mathrm{~mA}$. The input voltage limit is $5 \mathrm{~V}$, which gives a minimum input impedance of $20 \Omega$. This is the value used for $Z_{\mathrm{c}}(s)$. Having found $Z_{\mathrm{c}}(s)$, we can next find the value for $C$. The receiver impedance is the TLT impedance of (1) added to the impedance of the coupling capacitance $1 / s C$. For transmission line impedance matching, the impedance of the line must be matched to the impedance of the load to ensure maximum power deliver. Thus, $Z_{\mathrm{t}}(s)$ is equal to the impedance of the chosen communication medium ( $75 \Omega$ in our case). Setting the impedance of the coupling capacitor and receiver equal to $75 \Omega$ the only unknown entity is the capacitance value $C$. The parameters for our system were specified using this method and are presented in Table I.

\section{Experimental Evaluation}

Using the coupling circuit with the aforementioned parameter values and the prototype apparatus, we conducted experiments to demonstrate the feasibility of the method.

Fig. 10 shows a source digital signal before modulation and transmission, and the demodulated signal received by one of the nodes connected to the CPS lines. Here, the pulse interval is $10 \mu \mathrm{s}$, and the transmission rate is $100 \mathrm{~kb} / \mathrm{s}$. Although the highfrequency noise of the power supply is visible in the received signal (Fig. 10), the SNR of 18.1 ensures that the data is correctly 


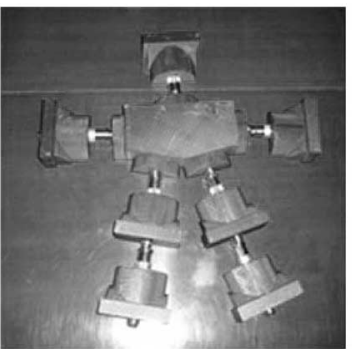

(a)

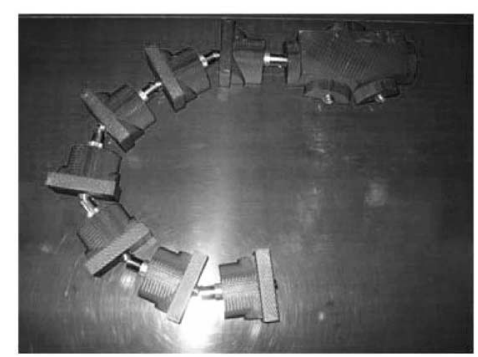

(b)
Fig. 11. Modular reconfigurable robot utilizing the dc PLC technology.

decoded. The $50 \%$ voltage gain confirms the predicted value of gain depicted in Fig. 8(B).

\section{Applications to Modular Robotics}

We now discuss the application of this technology to modular reconfigurable robotics. As the name implies, modular reconfigurable robots are distributed robotic systems in which multiple individual, independent modules can physically reconfigure themselves to perform a specified task. Such robotic systems have the advantage of adapting their physical structure to varying tasks determined by their environment [15]. These systems work well on tasks with unpredictable missions and geography, such as space exploration. Further, these systems, due to the multiplicity of components, are less susceptible to system-wide failure. The multiplicity of components also helps to reduce overall cost by allowing for mass production of components [16].

The multiplicity is also a source of problems - the utility of these systems depends heavily on the ability of individual modules to easily and conveniently connect to one another [15]. The modules must be physically connected in order to form the overall robot structure and to pass data to one another. Applying our dc PLC system to modular reconfigurable systems can greatly simplify the design of this physical connection by requiring only two electrical contacts between adjacent units.

For instance, Xerox PARC's PolyBot has a mechanism for establishing electrical connection between two modules. The robots use a four times redundant set of electrical connectors; on each module, there is a face with four sets of identical connectors located $90^{\circ}$ from one another. Thus, assuming that two modules are face-to-face, the required amount of rotation is always less than $90^{\circ}$. Nonetheless, there must be sufficient actuation to facilitate this rotation. However, with our dc PLC technique, the need for this redundancy is eliminated. A single concentric connector (such as a coaxial cable) at the center of each connection face is adequate to ensure power and data transmission using our dc PLC technique.

Fig. 11 depicts a modular reconfigurable robot utilizing our dc PLC transmission system in two configurations. The modular reconfigurable robot consists of a trunk, two legs, two arms, and a head, but can take many shapes, as indicted by the figure. The connection used in this case is a coaxial cable. Each of the "extremities" can be connected to any of the connection ports on the trunk. In this case, a dedicated power source on the trunk is used to provide power to the various limbs and simultaneously supply them with information necessary for reconfiguration of the individual modules. The dc PLC modem on each limb can then extract the command information and the power from the coaxial CPS line.

This is but one of many possible applications to robotics for which our dc PLC system shows promise. Specifically, the idea of reducing the amount of cabling means tangible savings in the weight and bulk of robotic systems.

\section{CONCLUSION}

In this paper, a dc power line communication system is presented. The technical issues specific to the use of a dc source are discussed and used to form functional requirements for a high-fanout system. An experimental apparatus confirmed our derived system model, and provided information on the system performance as the number of nodes in the system goes to infinity. By incorporating a TLT into the modem, we are able to exploit the resonance of the parasitics to ensure that power is delivered to the receivers, even for very high fanout. An example of how we can take advantage of this feature to broadcast to large numbers of receivers is presented as well. It has been demonstrated that this protocol will work for communication with multiple actuators connected to the line.

\section{APPENDIX A \\ DERIVATION OF $Z_{\mathrm{t}}(s)$}

For this derivation, we use Fig. 4. Following the procedure illustrated in [12, pp. 443-444], we can write

$$
\begin{aligned}
V_{1}(s) & =s L_{\mathrm{w}} I_{1}(s) \\
V_{2}(s) & =s L_{\mathrm{w}} I_{2}(s) \\
V_{\mathrm{g}, \text { out }}(s) & =V_{1}(s)+V_{2}(s) \\
I_{\mathrm{g}, \text { in }}(s) & =I_{1}(s)+\frac{V_{1}(s)}{R_{\mathrm{w}}}-I_{s}(s) \\
I_{\mathrm{g}, \text { in }}(s) & =I_{2}(s)+\frac{V_{2}(s)}{R_{\mathrm{w}}}-I_{s}(s) \\
V_{\mathrm{g}, \text { in }}(s) & =V_{1}(s)+V_{\mathrm{g}, \text { out }}(s)+V_{2}(s) .
\end{aligned}
$$

Combining these equations, we can write

$V_{\mathrm{g}, \text { in }}(s)=V_{1}(s)+V_{1}(s)+V_{2}(s)+V_{2}(s)=2\left(V_{1}(s)+V_{2}(s)\right)$

$$
I_{\mathrm{g}, \text { in }}(s)=\frac{1}{2}\left(\frac{V_{1}(s)+V_{2}(s)}{2 s L_{\mathrm{w}}}+\frac{V_{1}(s)+V_{2}(s)}{2 R_{\mathrm{w}}}+I_{\mathrm{g}, \text { out }}(s)\right) .
$$

Next, we can write

$\frac{V_{\mathrm{g}}(s)}{I_{\mathrm{g}}(s)}=\left(\frac{8 R_{\mathrm{w}} Z_{\mathrm{g}, \text { out }}}{Z_{\mathrm{g}, \text { out }}+2 R_{\mathrm{w}}}\right) \frac{s}{s+\frac{1}{L_{\mathrm{w}}}\left(\frac{R_{\mathrm{w}} Z_{\mathrm{g}, \text { out }}}{Z_{\mathrm{g}, \text { out }}+2 R_{\mathrm{w}}}\right)}$. 
Finally, we note that the magnetizing inductance $L_{\mathrm{m}}$, is equal to $2 L_{\mathrm{w}}$. So, we can finally write the impedance as

$$
Z_{\mathrm{t}}(s)=\frac{V_{\mathrm{g}, \text { in }}(s)}{I_{\mathrm{g}, \text { in }}(s)}=\left(\frac{8 R_{\mathrm{w}} Z_{\mathrm{l}}}{Z_{\mathrm{l}}+2 R_{\mathrm{w}}}\right) \frac{s}{s+\left[\frac{2}{L_{\mathrm{m}}}\left(\frac{R_{\mathrm{w}} Z_{\mathrm{l}}}{Z_{\mathrm{l}}+2 R_{\mathrm{w}}}\right)\right]} .
$$

\section{APPENDIX B IMPEDANCE COEFFICIENTS}

$$
\begin{aligned}
& \alpha=\left(\frac{R}{R+8 R_{\mathrm{w}}}\right), \quad \tau_{1}=R_{\mathrm{w}} C, \quad \tau_{2}=\sqrt{L_{\mathrm{m}} C} \\
& A=16 \alpha \tau_{1} \tau_{2}^{2} \\
& B=8(n+1)\left(R_{0}\right)\left(\tau_{2}^{2}\right) \equiv b_{1} n+b \\
& C=16 \alpha(n+1)\left(R_{0}\right)\left(\tau_{1}\right) \equiv c_{1} n+c_{2} \\
& D=2(n+\alpha)\left(\tau_{1}\right)\left(\tau_{2}^{2}\right) \equiv d_{1} n+d_{2} \\
& E=(n+1)\left(4 \alpha \tau_{1}^{2}+\tau_{2}^{2}\right) \equiv \varepsilon_{1} n+\varepsilon_{2} \\
& F=2(\alpha+1)(n+1)\left(\tau_{1}\right) \equiv f_{1} n+f_{2} \\
& G=2 \alpha(n+1)\left(\frac{\tau_{1}}{\tau_{2}}\right)^{2} \equiv g_{1} n+g_{2} .
\end{aligned}
$$

\section{APPENDIX C}

\section{GAIN COEFFICIENTS}

$$
\begin{aligned}
\alpha & =32 R_{0}^{3} L^{2} C \\
\delta & =4 R_{0}^{2} L^{2} C\left[9 n R_{\mathrm{s}}+R_{\mathrm{s}}+8 R_{0}\right] \\
\varepsilon & =2 R_{0} L(n+1)\left[4 R_{0}^{2} R_{\mathrm{s}} C+9 R_{\mathrm{s}} L+72 R_{0} L\right] \\
\xi & =40(n+1)\left(R_{0}^{2} R_{\mathrm{s}} L\right) \\
\eta & =8 R_{\mathrm{s}} R_{0}^{3}(n+1)
\end{aligned}
$$

\section{REFERENCES}

[1] A. Graf. (1996). SIPMOS high-current switches in automotive electronics: Extra features cut system costs, Components XXXI No. 5. [Online]. Available: http://www.infineon.com/cmc_upload/migrated_files/ document_files/Application_Notes/graf2.pdf

[2] P. L. Borrill, "MicroStandards special feature: A comparison of 32-bit buses," Microprocess. Microsyst., vol. 5, no. 6, pp. 94-100, Dec. 1985.

[3] D. Hines. (2000, Apr.). Unlocking the potential of power distribution networks. Powerline Economics. [Online]. Available: http://powerline.ipcf. org/doc/Power_Economics_-_Unlocking_The_Potential.pdf

[4] D. Strassberg. (1996, Jun.). Powerline communication: Wireless technology. EDN Mag. pp. 71-78.

[5] W. Downey and P. Sutterlin. (2001). Power line communication technology update. Echelon Corp. Presentation. [Online]. Available: http://www. echelon.com

[6] M. Tanaka, "High frequency noise power spectrum, impedance and transmission loss of power line in Japan on intra-building power line communications," IEEE Trans. Consum. Electron., vol. 34, no. 2, pp. 321-326, May 1998.
[7] J. E. Newbury, "Communications services using the low voltage power distribution network," in Proc. IEEE/PES Transmiss. Distrib. Conf. Expo. Oct.28-Nov. 2, 2001, vol. 2, pp. 638-640.

[8] Y.-F. Chen and T.-D. Chieuh, "A 100-kbps power line modem for household applications," in Proc. Int. Symp. VLSI Technol., Syst., Appl., Jun. 1999, pp. 179-182.

[9] R. A. Bigler and P. P. Bigler, "Integrated dc servo motor and controller," U.S. Patent 591 241, Jun. 1999.

[10] P. H. Sutterlin, "Powerline coupling network," U.S. Patent 5485 040, Jan. 1996.

[11] J. Sevick, Transmission Line Transformers, 4th ed. Atlanta, GA: Noble, 1996, pp. 1-1-3-15.

[12] C. C. Kuo, M. Y. Kuo, and M. S. Kuo, "Modeling and analysis of wideband power transmission line transformers," in Proc. 11 th Annu. Appl. Power Electron. Conf. Expo. Mar. 3-7, 1996, vol. 1, pp. 441-446.

[13] H. Meng, Y. L. Guan, and S. Chen, "Modeling and analysis of noise effects on broadband power line communications," IEEE Trans. Power Del., vol. 20, no. 2, pp. 630-637, Apr. 2005.

[14] A. Hussain, D. R. Campbell, and T. J. Moir, "Multi-sensor sub-band adaptive noise cancellation for speech enhancement in an automobile environment," in Proc. Adaptive Signal Process. Mobile Commun. Syst. (ref. 1997/383), IEE Colloq., Oct. 29, 1997, pp. 5/1-5/7.

[15] M. Yim, Y. Zhang, and D. Duff, "Modular robots," IEEE Spectr., vol. 39, no. 2, pp. 30-34, Feb. 2002.

[16] C. Unsal and P. K. Khosla, "Mechatronic design of a modular selfreconfiguring robotic system," in Proc. IEEE Int. Conf. Robot. Autom. Apr. 24-28, 2000, vol. 2, pp. 1742-1747.

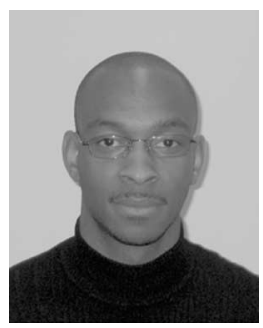

Eric R. Wade (S'04) received the B.S. degree in mechanical engineering, and the dual M.S. degrees in mechanical and electrical engineering and computer science from the Massachusetts Institute of Technology (MIT), Cambridge, in 2000 and 2004, respectively, where he is currently working toward the Ph.D. degree in mechanical engineering.

His current research interests include power electronics, servo-system design, digital communication, wearable sensing, circuit design, and system control.

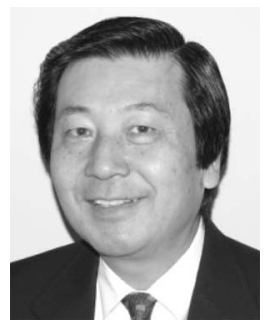

Haruhiko Harry Asada (A'85-M'05) received the B.S., M.S., and Ph.D. degrees, all in precision engineering from Kyoto University, Kyoto, Japan, in 1973, 1975, and 1979, respectively.

$\mathrm{He}$ is a Ford Professor of Mechanical Engineering and the Director of the Brit and Alex d'Arbeloff Laboratory for Information Systems and Technology in the Department of Mechanical Engineering at the Massachusetts Institute of Technology, Cambridge. His research has been concerned with robotics, biomedical engineering, and system dynamics and control. His current research interests include actuators, vast DOF robotic systems, and broadcast feedback. His biomedical research interests include wearable health monitoring and robotic aids for bedridden patients.

Dr. Asada is a Fellow of the American Society of Mechanical Engineers (ASME). He was the recipient of the Best Paper Awards at the IEEE International Conference on Robotics and Automation, in 1993, 1997, and 1999, and the Dynamic Systems and Control Outstanding Researcher Award from the ASME, in 1998. 